\title{
Théoriser le genre pour déjouer ses frontières et construire le sens
}

Julien Longhi et Nathalie Garric

\section{(2) OpenEdition}

Journals

\section{Édition électronique}

URL : http://journals.openedition.org/pratiques/3543

DOI : $10.4000 /$ pratiques.3543

ISSN : 2425-2042

\section{Éditeur}

Centre de recherche sur les médiations (CREM)

\section{Édition imprimée}

Date de publication : 1 juin 2013

Pagination : 31-46

Référence électronique

Julien Longhi et Nathalie Garric, «Théoriser le genre pour déjouer ses frontières et construire le sens », Pratiques [En ligne], 157-158 | 2013, mis en ligne le 18 décembre 2017, consulté le 08 mai 2019. URL : http://journals.openedition.org/pratiques/3543; DOI : 10.4000/pratiques.3543

(c) Tous droits réservés 


\title{
Théoriser le genre pour déjouer ses frontières et construire le sens
}

\author{
Julien Longhi \\ CRTF (EA 1392), Université de Cergy-Pontoise \\ Nathalie Garric \\ LLL (UMR 7270), Université de Tours
}

L'analyse de discours (désormais AD), depuis ses origines, nourrit le projet d'une sémantique discursive ou d'une théorie sémantique en rupture avec les sémantiques logico-grammaticales qui enferment le sens dans une intériorité à la langue et/ou se laissent prendre au piège de la spéculation et de l'illusion. Si ce questionnement sémantique n'a pas initialement théorisé la notion de genre, il a pourtant rapidement introduit dans la réflexion l'idée de frontière. En effet, selon Guilhaumou, Maldidier et Robin (1994), «Le sens n'est jamais posé par rapport à un extérieur non langagier; il se bâtit à travers des dispositifs d'archive où se manifeste la matérialité de la langue » mais, il est également « frontière et subversion de la frontière, négociation entre des lieux de stabilisation de la parole et des forces qui excèdent toute localité » (Maingueneau 2005). Des espaces de régulation langagière, clos et ouverts à la fois, sont définis par les auteurs comme partie prenante du sens. Dans cette contribution, notre propos sera d'éclairer la problématique des genres de discours du point de vue de l'analyse de discours en prenant appui sur des productions qui (se) jouent de la frontière entre genre politique et genre publicitaire. Nous effectuerons d'abord un retour sur certaines notions fondatrices de l'AD introduites en vue de saisir les espaces de normativité discursifs mentionnés que nous illustrerons d'un premier exemple publicitaire articulé à l'actualité politique du moment. Nous introduirons la notion de frontière en vue d'une théorisation dynamique du fonctionnement générique du discours. Nous mettrons ensuite en œuvre par le traitement d'un nouvel exemple, envisagé comme « moment discursif » (Moirand 2007), les considérations précédentes. Il s'agira de les éprouver et de montrer comment elles peuvent être repensées dans leur articulation à des apports moins « traditionnels » dans la discipline, mais féconds. 


\section{S'extraire de la contrainte générique pour analyser dans l'interdiscours}

L'AD a longtemps privilégié l'étude du discours, et l'analyse de texte, en développant ses analyses à partir de productions qui présentent une homogénéité souvent garantie par le choix de conditions de production identiques, lors de 1'opération de constitution du corpus. Ainsi enfermées dans une pratique a priori, les études ont favorisé l'identification de régularités textuelles propres à certaines pratiques socio-discursives, le discours politique ou le discours publicitaire, par exemple. Par cette méthodologie, elles ont toutefois neutralisé certains phénomènes propres au fonctionnement discursif et n'ont pas permis de définir une théorie du genre dynamique et non inscrite dans une visée typologique. Adam et Heidmann (2004), lorsqu'ils introduisent les notions de généricité et d'effet de généricité, abordent explicitement ces considérations :

«[...] il est “moins question d'examiner l'appartenance générique d'un texte que de mettre à jour les tensions génériques qui l'informent. Ce déplacement du genre à la généricité met en suspens toute visée typologique [et] permet de contourner l'écueil essentialiste" (Dion et al. 2004 : 17). Il s'agit d'aborder le problème du genre moins comme l'examen des caractéristiques d'une catégorie de textes que comme la prise en compte et la mise en évidence d'un processus dynamique de travail sur les orientations génériques des énoncés (2009: 13). [...] La prise en compte de cette hétérogénéité générique est, selon nous, le seul moyen d'approcher la complexité de la procédure qui relie un texte à l'interdiscours d'une formation sociale donnée » $(2009: 14)$.

En nous intéressant à des conflits discursifs du débat public, naissant dans l'interdiscursivité, mais présupposant simultanément des espaces génériques distincts, nous visons l'objectif défini par les auteurs. La notion de frontière est alors introduite pour souligner le caractère stable et dynamique à la fois, « flou et systémique » (ibid. : 18) à la fois des genres.

Prenons, à titre d'illustration préalable, la publicité pour la marque Porsche diffusée à l'issue de la publication médiatique d'une photographie montrant le potentiel candidat à la présidentielle, D. Strauss-Kahn, utilisateur du véhicule.

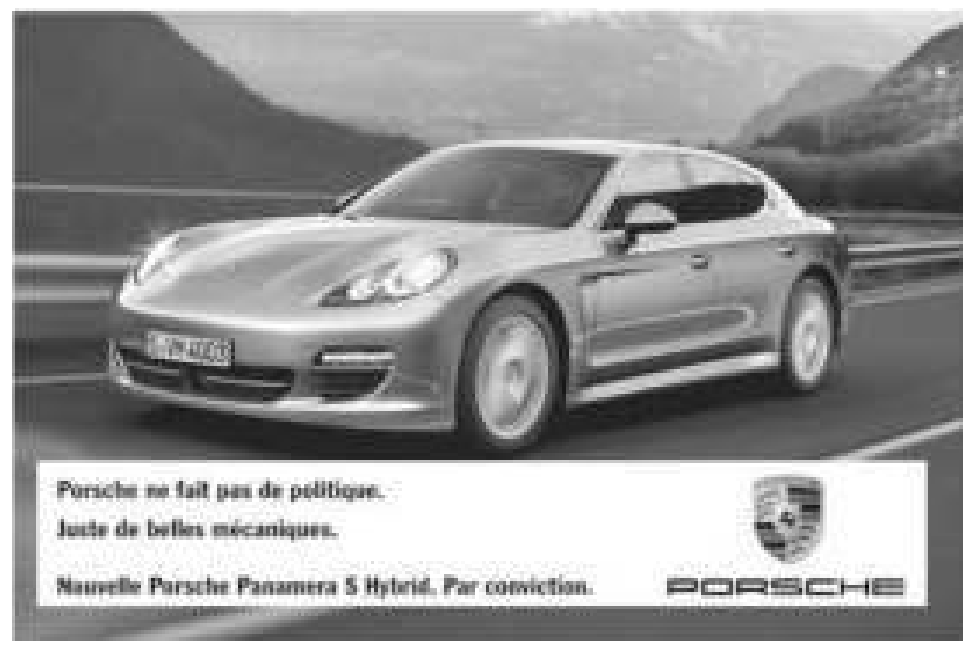


L'actualisation du genre publicitaire est explicite. On soulignera, par exemple, la présence des éléments constitutifs de toute publicité : une mise en scène iconique du produit, la présence d'un énoncé apparenté à une accroche, la mention associée de la marque et du logotype, enfin, l'occurrence d'un énoncé descriptif fonctionnant comme slogan. Chacun de ces éléments s'inscrit dans la valorisation de la voiture, définie comme objet de luxe alliant l'esthétique et la fiabilité et construite en auxiliaire de quête pour le consommateur (Charaudeau 1982). Pourtant, comme nous le montrerons progressivement, certains indices linguistiques détournent les attendus du genre affaiblissant sa frontière pour l'ouvrir à un autre genre, celui du discours politique.

\section{Théoriser les espaces de normativité}

Les analyses de discours ont toujours formulé l'hypothèse de différents espaces de structuration de la réalité discursive — internes, externes, internes/externes avec une hiérarchisation possible de chacun. Le genre n'existe pas de façon absolue, il entre en relation avec d'autres notions.

\subsection{Des notions clés : formation discursive, interdiscours et genre}

Ces espaces sont historiquement et traditionnellement traités en termes de formation discursive, d'interdiscours et de genre. Nous rappelons de manière synthétique dans ce qui suit ces notions pour ensuite les compléter par des approches qui redéfinissent les genres comme des catégories dynamiques.

Foucault introduit la notion de formation discursive pour identifier " Un ensemble de règles anonymes, historiques, toujours déterminées dans le temps et dans l'espace qui ont défini à une époque donnée, et pour une aire sociale, économique, géographique ou linguistique donnée, les conditions d'exercice de la fonction énonciative » (Foucault $1969: 153$ ). Pêcheux la reprend, l'inscrit dans l'AD et l'articule à l'espace socio-idéologique l'exposant ainsi à de nombreuses remises en cause ultérieures : «Nous appellerons dès lors formation discursive ce qui, dans une formation idéologique donnée, c'est-à-dire à partir d'une position donnée dans une conjoncture donnée déterminée par l'état de la lutte des classes, détermine "ce qui peut et doit être dit" (articulé sous la forme d'une harangue, d'un sermon, d'un pamphlet, d'un exposé, d'un programme, etc.) » (Pêcheux 1975 : 144). Alors que Foucault reliait la formation discursive à l'existence de contraintes qui déterminent les positions énonciatives, Pêcheux la relie très explicitement à l'idéologie, à une position de classe dominante qui relègue les formes linguistiques, non à des pratiques à proprement parler, mais à des manifestations de surface quasi-secondaires dans le conflit idéologique. Malgré les différences notionnelles, il est notable que la formation discursive dispose d'incidences linguistiques. D'ailleurs, Beacco (2004 : 116) propose d'utiliser le concept de genre, selon une conception proche de celle de Pêcheux, afin «d'articuler des formes discursives à des lieux, définis de manière plus large que par les dimensions de la situation d'énonciation, qui est un construit de nature linguistique, sans dimensions sociales ».

Par rapport à ces espaces clos et «frontiérisés », initialement introduits dans le premier modèle de Pêcheux, la notion d'interdiscours constitue un moyen théorique d'une part pour maintenir la pertinence des formations discursives, d'autre part pour rendre compte de leur interaction et de leurs rapports de force. Elle est 
en ce sens une procédure de « défrontiérisation » des formations discursives, qui deviennent alors « ouvertes, perméables et hétérogènes » (Krieg-Planque 2004). L'interdiscours est le lieu de construction de préconstruits, déterminations idéologiques antérieures et indépendantes, qui trouvent des manifestations intradiscursives telles que la relative ou la nomination. L'idéologie précède donc la pratique discursive et sa potentielle intrication avec d'autres formes de déterminisme, la langue ou encore les effets de généricité, n'est pas envisagée dans ce cadre. C'est pourtant en termes de manifestations interdiscursives que peut être analysée, dans l'exemple de la publicité pour Porsche, l'occurrence de la négation dans le contexte de l'adverbe d'énonciation « juste ». Elle introduit un mouvement argumentatif polyphonique qui construit la voix (ou le point de vue) de l'instance accusatrice. Selon Leeman (2004), cet adverbe, intervenant dans un « contexte de dénégation », est employé pour « contrecarrer d'avance ce que l'interlocuteur peut penser » et pour l'amener ainsi vers une conclusion plus favorable que celle qui semblait s'imposer. La négation et l'adverbe sont des manifestations intradiscursives du préconstruit, Porsche fait de la politique. Ils bloquent les inférences auxquelles pourrait donner lieu cette assertion, susceptibles d'établir des intérêts entre les acteurs évoqués.

$\rightarrow$ Contrairement à ce que vous croyez, Porsche ne fait pas de la politique.

$\rightarrow$ Contrairement à ce que vous croyez, Porsche ne fait que de belles mécaniques.

Ce sont bien ici des conflictualités idéologiques qui s'expriment : situées dans l'espace du débat public, elles confrontent les voix de la formation discursive socialiste à celles de ses opposants.

La notion de genre est absente des travaux de Pêcheux. Développée notamment par Bakhtine, elle relève également d'une procédure de «frontiérisation » des pratiques discursives. Indépendante de la notion d'idéologie, elle vise à qualifier les productions discursives en fonction de régularités linguistiques qui résultent de règles et de contraintes propres à certaines situations de production et/ou d'énonciation.

"L'utilisation de la langue s'effectue sous forme d'énoncés concrets, uniques (oraux ou écrits) qui émanent des représentants de tel ou tel domaine de l'activité humaine. L'énoncé reflète les conditions spécifiques et les finalités de chacun des domaines, non seulement par son contenu (thématique) et son style de langue, autrement dit par la sélection opérée dans les moyens de la langue - moyens lexicaux, phraséologiques et grammaticaux -, mais aussi et surtout par sa construction compositionnelle. Ces trois éléments [...] fusionnent indissolublement dans le tout que constitue l'énoncé, et chacun d'eux est marqué par la spécificité de l'échange. [...] chaque sphère d'utilisation de la langue élabore ses types relativement stables d'énoncés, et c'est ce que nous appelons les genres de discours. » (Bakhtine, $1984: 265$ ).

Ainsi, bien que le message de la marque Porsche soit inscrit sans ambiguïté dans le genre publicitaire, la mention directe de l'univers politique, l'occurrence de la négation, décalée dans ce genre relevant par définition d'une rhétorique de l'éloge (Adam \& Bonhomme 1997), ainsi que le syntagme «par conviction », propre à la pratique politique et en discordance avec les valeurs esthético-hédoniques de la publicité, actualisent les spécificités du genre politique. Par exemple, hors 
de son contexte générique, la référence à la conviction actualise pour le politicien une qualification par son complémentaire linguistique : DSK fait de la politique sans conviction ; il en fait pour des raisons financières. L'existence d'espaces normatifs d'interprétation est donc à la fois construite et déconstruite, permettant simultanément de disqualifier D. Strauss-Kahn en dénonçant son mode de vie et ses convictions et de valoriser la marque Porsche par la prédication valorisante restrictive.

Il est ici nécessaire d'insister sur la notion de finalité introduite supra par Bakhtine. Elle implique que la caractérisation générique requiert des catégories spécifiques - catégories discursives - construites dans la totalité textuelle et qui ne peuvent être réduites aux « seules catégories sémantico-grammaticales des problématiques énonciatives indicielles » (Moirand 1992:30). Ces catégories s'appuient sur des indices repérables dans la matérialité textuelle, mais relèvent d'une construction de l'analyste par la reconnaissance de cohérences fonctionnelles établies par les unités. Là encore finalité ou fonctionnalité ne sont pas des notions simples : Moirand distingue en effet les «fonctions » qui renvoient à une « intentionnalité des discours » et les «schématisations » qui s'appuient sur un « projet argumentatif du locuteur » établi pour un auditoire en fonction d'une évaluation de la situation. Ainsi, peut-on opposer les catégories de la langue identifiées par Charaudeau (1992) sur la base d'une intention de communication (actualisation, qualification, désignation par exemple) et les modes d'organisation du discours (énonciatif, descriptif, narratif, argumentatif). L'approche textuelle d'Adam retranscrit également cette distinction entre une forme de portée communicative et pragmatique du texte, et une compositionnalité fonctionnelle. Il distingue ainsi des empaquetages propositionnels (proto)typés que sont les « séquences » — c'est le cas lorsque les regroupements de propositions sont réglées par les cinq grands types des séquences de base suivants : narrative, descriptive, argumentative, explicative, dialogale — et des « unités configurationnelles ». Si « Un texte est, d'une part, une suite linéaire de parties (paragraphes correspondant à des périodes ou des séquences) formant une structure compositionnelle donnée » (Adam, 2005 : 68), un texte est, d'autre part, un tout de sens sémantique ou pragmatique, une unité configurationnelle.

Selon une distinction introduite par Maingueneau (2005), le genre renvoie aux unités dites topiques dans le sens où elles correspondent à un prédécoupage des pratiques verbales, alors que les formations discursives relèvent des unités dites non topiques : elles sont construites par le chercheur indépendamment de frontières préétablies, leur rôle étant de dépasser les frontières en se situant dans l'interdiscours, notamment sur des événements discursifs. Cette distinction introduite, l'auteur souligne qu'il ne peut y avoir d'AD sans unités topiques, mais qu'il faut aussi prendre en compte, à l'aide de la notion d'interdiscours, ce qui déjoue les frontières. Dans le cadre des unités topiques, l'auteur identifie des « unités domaniales » qui donnent lieu à des «types de discours » susceptibles de correspondre à un même appareil institutionnel, tels que le discours publicitaire et le discours politique, ou à un même positionnement, tel que le discours socialiste, par exemple. Dans la terminologie de l'auteur, ces types de discours peuvent trouver plusieurs «genres de discours » qui correspondent à des dispositifs communicationnels : par exemple la publicité magazine pourra être distinguée de la publicité par affichage de rue. Les unités topiques recouvrent également des « unités transverses » qui peuvent recevoir une conceptualisation linguistique, fonctionnelle - discours 
propagandiste, par exemple — ou encore communicationnelle — le discours de vulgarisation, par exemple.

Rappelons que discours politique et discours publicitaire sont traditionnellement identifiés comme deux genres distincts reliés à deux formations socio-discursives. Ils divergent par des attendus langagiers singuliers, notamment fonctionnels, et ce malgré des régularités partagées qui les unissent dans le discours propagandiste (Garric \& Léglise 2005). Selon Charaudeau (1984), la propagande est « un discours de manipulation (au sens sémiotique) qui consiste à faire croire au destinataire d'une part qu'il a un manque (qui repose sur des valeurs éthiques ou hédoniques), d'autre part que le moyen qui lui est proposé pour combler ce manque est le seul et le meilleur», mais alors que le premier est centré sur « la dénonciation du mal », le second s'inscrit dans « la révélation du bien » (Gourevitch 1981). La publicité analysée joue de ces différences de finalité propres aux unités topiques, elle les met en conflit, révélé par l'hétérogénéité (Authiez-Revuz 1982), et exprime simultanément des positionnements idéologiques propres aux formations discursives politiques qui déterminent ce qui peut être dit ou ne pas être dit, ou encore ne pas être fait (s'afficher utilisateur d'un véhicule de luxe) dans l'espace topique du discours socialiste.

Dans le cadre de la Sociologie du langage, Achard, à l'aide de la notion de registre, peut nous permettre de compléter cette analyse. Il distingue trois notions :

« Une formation discursive est la structuration de l'espace social par différenciation des discours. Cette différenciation repose sur des accumulations de "textes" dans un même voisinage, ce que j'appellerai registre discursif » (Achard 1995, 84). « On distinguera l'organisation institutionnelle et sociale des formations (que nous appelons "registres discursifs") et l'organisation par les formes (que nous appelons "genres")» (Achard $1996: 12$ ).

Le genre est caractérisé par un ensemble de régularités linguistiques, résultant des contraintes socio-institutionnelles des registres discursifs. Un registre est lié à une place sociale manifestée par des énoncés partageant une même indexicalité. Il est une hypothèse, non un a priori idéologique, en lien avec des régularités formelles mais sans recouvrement générique systématique : «La dynamique des écarts est le phénomène central sur lequel s'articule le linguistique et le social» (1988). Un registre en outre peut illustrer plusieurs effets de généricité. Le rapport entre forme et pratique sociale repose sur un lien de motivation qui ne nie toutefois pas une certaine indépendance de la première vis-à-vis du registre. Il est dès lors acceptable de poser — même s'il reste à définir — un déterminisme linguistique. Il devient exclu que le texte soit un reflet d'une extériorité puisque le registre s'intercale en quelque sorte entre ces deux extrémités du tout discursif.

$\mathrm{Au}$ cours de ce parcours épistémologique, nous avons identifié une pluralité d'emplois des notions qui s'articulent au concept de genre, lui-même pluriel, et qui contribuent à sa problématisation discursive. Pour clarifier les usages mentionnés, propres parfois à des auteurs distincts, nous proposons de les recenser dans le tableau suivant ${ }^{(1)}$ :

(1) Le caractère synthétique de ce tableau oblige à une introduction simplifiée et parfois radicalisée des différentes notions manipulées par les auteurs convoqués qui ont pu dans 1'évolution de leur réflexion relativiser les positions globalisantes que nous restituons. 


\begin{tabular}{|l|l|l|l|}
\hline $\begin{array}{l}\text { Approche } \\
\text { centrée sur }\end{array}$ & Auteurs & Notions opérantes & Portée pour le genre \\
\hline L'énoncé & Bakhtine & $\begin{array}{l}\text { Genre / Pratiques } \\
\text { socio-discursive }\end{array}$ & $\begin{array}{l}\text { Caractérisation formelle } \\
\text { multiniveaux }\end{array}$ \\
\hline L'idéologie & Pêcheux & Interdiscours & $\begin{array}{l}\text { Animation des formations } \\
\text { discursives }\end{array}$ \\
\hline Le texte & Adam & (Effet de) Généricité & $\begin{array}{l}\text { Transgénéricité des textes et } \\
\text { transversalité générique des genres, } \\
\text { rupture avec les typologies a priori }\end{array}$ \\
\hline Le discours & Maingueneau & $\begin{array}{l}\text { Unités topiques / } \\
\text { unités non topiques } \\
\text { Registre }\end{array}$ & $\begin{array}{l}\text { Hétérogénéisation des espaces } \\
\text { normatifs et défrontièration en } \\
\text { discours } \\
\text { Non isomorphisme idéologie/genre, } \\
\text { espaces de stabilité sociale et } \\
\text { formelle et dynamique des écarts }\end{array}$ \\
\hline
\end{tabular}

Portée de différentes théorisations discursives pour le concept de genre

\section{Construire et déconstruire les frontières : étude de cas}

Nous étudions, dans la suite de cette contribution, le jeu de frontière mis en évidence par une étude de cas inscrite dans l'actualité récente. D'un point de vue méthodologique, notre analyse consiste donc à rechercher dans l'interdiscours les processus de construction du sens, en prenant appui sur des productions langagières relatives à un «moment discursif» (Moirand 2007). L'introduction du concept de moment discursif, compris comme matérialisation historique dans l'espace public ou événement constitué par un grand nombre de productions discursives liées à un objet, permet « de refuser de poser a priori les paramètres sociaux d'une situation de communication globale, conçue bien souvent comme statique, alors qu' on voudrait saisir la complexité dynamique des circuits de la communication médiatique à travers son inscription dans la matérialité textuelle » (5).

Ainsi, en donnant à voir les échos et le dialogisme entre les discours relevant de genres différents mais de thèmes semblables, nous pouvons espérer repérer les différents espaces de normativité à l'œuvre. Partant du discours publicitaire qui convoque le jeunisme, le questionnement des frontières avec le discours politique permettra d'en saisir la manifestation qu'elle s'incarne dans des positionnements idéologiques ou des éléments de langage, si certains usages lexicaux ou sémantiques deviennent caractéristiques (voire définitoires) de discours. Afin de proposer une analyse unifiée des différentes dimensions, linguistiques, discursives, sociales et idéologiques, à l'œuvre dans le processus d'interprétation, nous proposons de mener cette seconde analyse à l'aide d'apports théoriques nouveaux empruntés à la pragmatique topique de Sarfati.

La publicité de Virgin Radio qui sert de point de départ à notre travail est a priori propre à un espace discursif, celui de la publicité par affichage de rue. Elle a pourtant déjoué ses frontières pour devenir dans l'espace politique et médiatique, peuplé de discours en relation avec la réforme des retraites, un objet discursif problématique : elle s'est ainsi attiré les foudres des médias et des politiciens, au point de se voir censurée par le maire socialiste de Clichy-La-Garenne. Le changement de discours a été accompagné d'une recontextualisation problématique dans 
le discours politique, dont les effets ont également modifié «l'écologie » du discours publicitaire. L'objet discursif de la publicité n'est plus le même, il est reconstruit : la chaîne Virgin Radio quitte la scène publicitaire, elle est subsumée par la question de la reforme des retraites.

\subsection{Le jeunisme publicitaire : du dicible à l'indicible}

Le 14 septembre 2010, la Radio Virgin diffuse par publicité de rue une série d'affiches publicitaires, construites sur le modèle suivant :

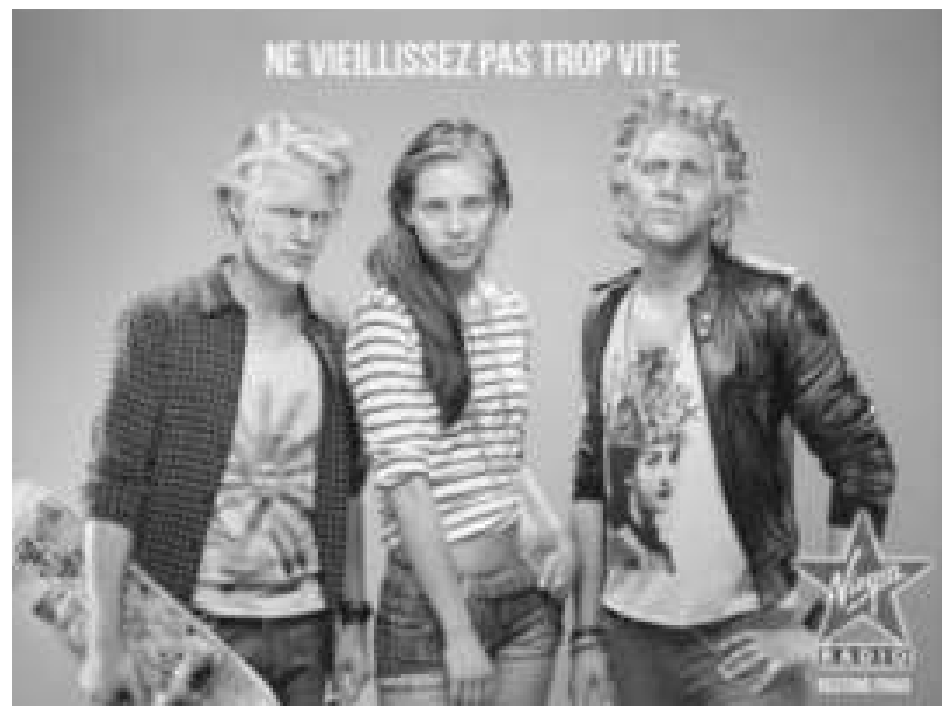

A priori, si on situe notre interprétation dans le cadre strict du genre discursif publicitaire, cette affiche relève d'une procédure de persuasion bien rodée. Exploitant le concept du « jeunisme », elle vise la séduction du potentiel consommateur par la définition du produit comme auxiliaire de jouvence, comme source de régénérescence. Cette publicité, à l'instar de nombreuses autres depuis plusieurs années, s'adresse à une cible senior qui, du fait de l'allongement de la durée de vie associé à une amélioration des conditions sanitaires et sociales, détient un pouvoir d'achat qui ne pouvait rester ignoré. Les personnes âgées vivent plus et surtout mieux, elles éprouvent de nouveaux besoins, qu'ils soient objectifs ou subjectifs. Dans ce contexte, le message publicitaire doit adapter sa promesse à ces consommateurs dont l'augmentation de l'espérance de vie trouve pour corollaire l'inquiétude de la dégradation au moins physique. L'argument de jeunisme, tel qu'il est construit dans le genre du discours publicitaire, est donc une forme de revalorisation du vieillissement par son retardement physique, moral ou intellectuel.

À l'intérieur de cet espace d'interprétation délimité par ce que nous savons du genre du discours publicitaire, nous identifions un message en conformité avec nos habitudes de consommateurs potentiels. Pourtant, déjà, certains indices suscitent l'étonnement par rapport à la majorité des publicités fonctionnant sur le concept du jeunisme. Elle ne semble pas respecter les ressources sémiotiques du jeunisme. Ses signifiants restent en général relativement discrets et consistent à gommer les effets de l'âge sur les visages ou à mettre les seniors en relation avec des activités dynamiques, tout en conservant des indices de l'âge effectif par des attri- 
buts physiques (des cheveux grisonnants) ou des indices symboliques (la présence d'enfants). Sur le plan linguistique, le discours publicitaire exploite l'expression du maintien de la jeunesse plutôt que celle d'une vieillesse retardée. Ainsi peut-on opposer les messages publicitaires de la marque Évian, par exemple, à celui de Virgin Radio :

— Boire chaque jour une eau pure et équilibrée entretient la jeunesse de votre corps. (Évian)

— Évian déclaré source de jeunesse par votre corps.

- Ne vieillissez pas trop vite. (Virgin Radio)

La publicité Virgin Radio contraste avec ce dispositif « habituel» : elle stigmatise le vieillissement par l'affichage impudique et caricatural de visages qui sont absents d'une part du discours publicitaire et d'autre part de toute publicisation non spécialisée, c'est-à-dire s'adressant exclusivement à la population senior. Ce dysfonctionnement par rapport aux attendus discursifs rend les frontières du genre poreuses. Il les ouvre à des sources d'inférences interprétatives, surtout si le concept du jeunisme est susceptible de rencontrer d'autres considérations relatives à l'âge dans l'espace interdiscursif. En effet, selon Maingueneau (2005), « Replier l'analyse du discours sur les seules unités topiques, ce serait dénier la réalité du discours, qui par nature met constamment en relation discours et interdiscours : l'interdiscours "travaille" le discours, qui en retour redistribue cet interdiscours qui le domine. La société est parcourue d'agrégats de paroles agissantes qu'on ne peut assigner à un lieu ». Seule cette attitude permet de comprendre pourquoi la publicité étudiée a été l'objet de controverses, jusqu'à recevoir la censure politique.

Nous formulons donc l'hypothèse que, construit aux frontières du registre publicitaire, le message Virgin Radio vient côtoyer celles du registre politique, animé par la réforme des retraites, arrière-plan qui occupe 1'espace public et qui participe à l'interprétation du message dans la dualité des positionnements de chaque parti politique. Dans ce contexte, la promesse d'une vieillesse qu'apparente dans un corps en pleine vitalité et apte à l'activité professionnelle ne peut pas s'énoncer naïvement comme en témoigne certains énoncés circulant dans l'espace médiatique. L'analyse des formes textuelles ne peut donc se concevoir indépendamment d'interprétants non inscrits dans le texte lui-même tout en étant constitutifs de la textualité. Ces formes ne sont en conséquence interprétables que dans 1'interaction, le jeu d'influence, qui anime les pratiques discursives et opacifie la linéarité textuelle.

\subsection{Analyser le conflit à l'aide de propositions de la Pragmatique topique}

Les notions d'institution de sens et de communauté de sens, empruntées à Sarfati, permettent de se saisir d'une partie de l'opérativité de celles de formation discursive et d'interdiscours chez Pêcheux, mais en les rattachant non plus à une position et à une idéologie, mais à une place spécifique dans une ou plusieurs institutions de sens et sans exclure l'existence de déterminations linguistiques. Sarfati (2008) indique qu'une institution de sens est un dispositif socio-discursif qui a pour finalité la production ainsi que la pérennisation de normes de pensée, d'expression et d'action auprès des acteurs sujets qui y participent. Une institution de sens coïncide avec un domaine de pratique, c'est-à-dire qu'elle se subdivise en 
communautés de sens. Une institution de sens produit son propre sens commun, et « une formation de sens commun est toujours le fait d'une institution de sens spécifique, et, incidemment, des variations que ses différentes normes subissent dans les communautés de sens qui en dépendent» (Sarfati $2010: 344)$.

Selon Sarfati, « tout sujet dispose d'une compétence topique. Elle est l'aptitude des sujets à sélectionner et identifier - à la production comme à la réception les topiques afférentes à une situation langagière donnée » (2002:112) et elle « permet de déterminer le profil normatif d'un discours ainsi que des séries textuelles qui lui sont afférentes » (2008: 100). Ainsi, si les topiques suscitées ne coïncident pas avec celles afférentes, le conflit patent à tout discours, puisque articulé à l'interdiscours, peut devenir l'objet même du discours, comme dans ce commentaire extrait du site du Parisien :

Je suis tout à fait d'accord que cette pub ne met pas en valeur l'âge mûr et qu'elle peut blesser beaucoup de gens qui ne veulent pas vieillir physiquement... la vieillesse physique n'a rien avoir avec ce qu'on a dans la tête de toute façon, et je connais bon nombre de jeunes ...très vieux! mais de là à en faire une histoire politique, c'est ridicule... il faut en rester au premier degré.

Cet exemple pointe en effet le fait que la perméabilité entre les discours n'est pas une évidence pour les acteurs du débat public et que le franchissement de cette frontière doit inclure le déplacement des seuils de construction du sens, en rapport avec les normes et sens communs qui ont cours dans les différents discours. La compétence topique d'une formation discursive s'ajuste au type de discours, et ici le « jeunisme » de la publicité n'impose pas, selon le locuteur, sa récupération en politique. Sa valeur publicitaire est cependant affaiblie par la porosité de la formation discursive publicitaire et l'actualisation simultanée qu'elle engendre de celle du discours politique. Ainsi le locuteur s'en remet-il à un travail métadiscursif destiné à bloquer l'intrusion de l'univers politique et l'interprétation qu'il suscite. Il réalise un travail de « frontiérisation » que d'autres discours peuvent déconstruire faisant entrer d'autres interprétants qui sont appuyés sur les propriétés elles-mêmes de la discursivité mais également sur certaines disponibilités sémantiques. C'est donc à partir de deux sens communs, issus de deux institutions de sens distinctes, que se négocie l'interprétation, en jouant sur la plasticité et le dynamisme des communautés de sens. Et c'est dans l'entrelacement de ces sens communs, propres aux communautés de sens issues des institutions de sens, que naît l'interprétation. Dans le prolongement de ces considérations, Sarfati propose de traiter les relations entre discours et idéologie d'une manière « inédite » : «Nous tenons qu'il n'y a pas de discours idéologique, mais seulement des usages idéologiques de certains discours. Autrement dit un discours ne devient idéologique ou ne reçoit le statut d'idéologie qu'au terme d'un processus d'idéologisation » (2011 : 157) qui « consiste précisément dans le “démarquage mimétique et stratégique" d'un discours donné » (2011 : 158).

La négociation métadiscursive est l'indice de l'enjeu d'idéologisation sous-jacent à cette publicité qui consiste pour une communauté de sens, inscrite dans l'institution publicitaire, à concevoir le jeunisme comme une réalité non problématique, puisqu'il est source de régénérescence, et pour une autre communauté de sens, inscrite dans l'interaction des institutions de sens, à concevoir le jeunisme comme une réalité problématique, puisqu'il se définit comme un handicap social d'une part et comme une cause de persévérance laborale d'autre part. L'interprétation polémique de cette publicité naît donc à la fois de fonctionnements discursifs 
stabilisés au sein d'espaces génériques et de fonctionnements interdiscursifs dynamiques, volatils, caractéristiques de la réalité du discours qui nie tout sens asocial et anhistorique.

La distinction entre doxa et idéologie introduite par Sarfati (2011) éclaire le conflit présenté par la publicité de Virgin Radio. Il s'agirait alors de distinguer doxa sur le jeunisme et idéologie du jeunisme, afin de repérer, à la production et à la réception, les éléments devenus problématiques et qui ont généré le conflit d'interprétation. L'idéologisation établie dans cette situation, et critiquée par le PS, se fonde sur une élaboration réfléchie, et est travaillée par les différents acteurs. Sarfati propose d'ailleurs d'identifier un continuum entre une doxa spontanée, aléatoire, une doxa à caractère idéologique obtenue par fait d'institution, et délibérée, et enfin l'idéologie qui est planifiée et réfléchie. Il est en outre notable que la doxa a une fonction phatique alors que l'idéologie a une fonction pratique. Aussi, concernant la publicité de Virgin Radio, la réception a transformé la nature du jeunisme, conçu probablement par les annonceurs comme une doxa (même à caractère idéologique, il ne devait pas être question de lui attribuer une fonction pratique), en idéologie. À partir d'une doxa sur le jeunisme liée à une institution de sens, existante et repérable par diverses attestations en discours, on passe à l'idéologie du jeunisme telle qu' elle est critiquée par les opposants à l'affiche de Virgin Radio. Aussi, cette conflictualité s'explique par le changement de statut sémiotique du jeunisme, montré non plus comme une élaboration spontanée mais comme une élaboration réfléchie et donc manifestée par des commentaires métadiscursifs. Finalement, le caractère polémique pourrait tenir au statut discursif du jeunisme : contestable mais faiblement générateur de conflit en tant que doxa, il devient un objet de controverse s'il accède au statut d'idéologie.

\subsection{Indices des conflictualités et de construction sémantique}

Nous avons montré ailleurs (Garric \& Longhi, à paraître) que les tensions liées à la campagne de Virgin Radio peuvent s'expliquer par le fonctionnement sémantique du terme « jeune ». Il témoigne d'une faille potentielle dans la mise en scène de cette publicité, qui, si elle est saisie dans l'interdiscursivité du moment discursif, ouvre l'interprétation du jeunisme à la conflictualité des espaces discursifs et de leurs topiques et doxa respectives.

Le fonctionnement sémantique de «jeune » procède de deux modalités différentes : son sens dans la publicité serait en effet proposé et perçu comme immédiatement argumentatif, alors qu'il serait revendiqué comme plus « référentiel » (dans un sens démographique) dans le discours politique. Le TLFI identifie en effet deux dimensions sémantiques dans le fonctionnement de l'adjectif « jeune ». Certains de ses sens sont relatifs à l'âge, signifiant « qui est peu avancé en âge », et sur le plan physique ou psychologique «Qui est celui, celle d'une personne peu avancée en âge ». Dans les autres sens, le jugement concernant l'âge est secondaire, et jeune peut caractériser une personne âgée ou une réalité ancienne. Dans ces cas, le terme renvoie à une image sociale valorisée de la jeunesse («vitalité et fraîcheur physique, dynamisme et spontanéité dans l'action, vivacité intellectuelle »), ou à une image morale défavorable de la jeunesse (" inexpérience et manque de maturité dans l'action, légèreté morale et intellectuelle »). Jeune peut aussi renvoyer à l'image sociale des jeunes gens particulière à une époque et à un espace discursif. Le latin rend d'ailleurs compte de cette dualité, avec jeune homme (adulescens) et homme jeune (juvenis). 
Les formes repérables dans le discours politique contemporain de cette campagne publicitaire semblent investir singulièrement ces formes de langue. Dans l'article « Pourquoi réformer les retraites?» publié le 13 octobre 2010 sur le site de l'Élysée par exemple, on trouve des expressions telles que « les personnes qui travaillent aujourd'hui », " les pensions des personnes aujourd'hui retraitées », "l les générations du baby-boom arrivent à l'âge de la retraite », " 4 actifs pour un retraité » ou encore dans, un article du 13 juin 2010, « Sauver notre système de retraites par répartition menacé par des déséquilibres », avec « la démographie française se caractérise par un vieillissement de la population, ce dont témoignent les départs à la retraite massifs des générations issues du baby-boom. On comptait 4 cotisants pour 1 retraité ».

Avec « personnes », « actifs » et « cotisants », le discours politique tente de neutraliser l'axiologisation pour éviter le caractère polémique : le terme « jeune » est peu employé dans ces discours centrés sur la question des retraites ; les considérations portées à l'âge sont actualisées par un lexique administratif évacuant les mentions physiques et psychologiques. Les préoccupations sur la réforme de la retraite génèrent une interprétation centrée sur le vieillisme, sur le vieillissement de la population en contraste avec la société active. Ce vieillisme pudique se présente comme une acceptation problématique dans laquelle «vieillir » s'interprète ou comme « devenir inactif» ou comme « rester actif plus longtemps ».

La porosité de la publicité étudiée ouvre le fonctionnement sémantique de ses unités à cette interprétation politique : le jeunisme publicitaire rencontre le vieillisme politicien. Ainsi l'accroche « $\mathrm{Ne}$ vieillissez pas trop vite » peut prendre une valeur axiologique performative négative - le terme « vieillir » étant luimême plus représentatif du discours politique que publicitaire. Le conseil devient quasiment une menace devant la nécessité de conserver, comme le mentionne le slogan, une certaine « fraîcheur ». « Restons frais » n'est pas en effet « restons jeune », il ne s'agit pas de préserver les attributs de la jeunesse ou du jeunisme, mais des atouts en quelques sortes sanitaires susceptibles de qualifier l'aptitude à l'activité professionnelle et à l'utilité sociale.

Cette publicité s'appuie donc sur le topos favorable de la jeunesse (définitoire du jeunisme), mais ce topos devient problématique dans l'interaction des constituants de cette publicité et de l'interdiscours du fait de disponibilités sémantiques offertes par le fonctionnement sémantique de "jeune ». C'est ici le même enjeu que celui identifié dans l'analyse de la publicité précédente, un enjeu d'idéologisation. Il ne procède toutefois pas de manière identique : alors que la publicité Porsche procède à un cadrage référentiel invitant au franchissement des frontières, la publicité Virgin Radio repose sur des indices plus discrets et surtout sur un processus de contextualisation (ou de cadrage) déterminé par l'actualité politique. Autrement dit, et même si certains composants sémiotiques sont génériquement décalés, c'est la propriété du discours en tant que trace discursive et support d'une hétérogénéité constitutive (Authier-Revuz 1982) qui détermine le décloisonnement générique qui vient investir spécifiquement les formes.

La publicité Porsche, fonctionnant en écho volontaire avec l'actualité, introduit des opérateurs pour ouvrir l'interprétation publicitaire. Certains reposent plus singulièrement sur des fonctionnements (ici argumentatifs) de langue, tels que « juste » associé à la négation, qui ne requièrent pas foncièrement le contexte large médiatico-politique (ou socio-historique). D'autres renvoient à des stabili- 
sations syntagmatiques attachées à des régularités génériques plus ou moins marquées et dont l'interprétation est construite dans le contraste discours publicitaire et discours politique, notamment pour la locution «par conviction ». Enfin, d'autres, telle que la mention cadrative, impliquent les discours en tant que pratiques stabilisées associées à des lieux de production et générant des représentations propres. Le message trouve ainsi des clefs interprétatives situées dans une autre pratique, mais ce faisant retrouve sa valeur sémiotique positive à l'intérieur du genre, dont les frontières sont ainsi réaffirmées. Le sens est construit dans l'interdiscursivité qui suppose l'existence de genres distincts, mais également, après l'ouverture des frontières, dans le retour aux espaces génériques où chaque objet discursif reçoit une valeur axiologique, valorisante pour l'un, dévalorisante pour l'autre. Un jeu de miroirs met ainsi en correspondance les interprétations propres à chaque discours, avec des dynamiques spécifiques qui s'appuient, à partir d'un même fonctionnement scalaire, sur un « haut degré » comparable sur les échelles de valeurs respectives à chaque genre, favorisant l'analogie entre l'intensité de valorisation attachée à Porsche et l'intensité de la critique attribuée à DSK.

\section{Les notions de genre et de frontière}

L'introduction de la notion de frontière dans la réflexion en vue d'une redécouverte de la notion de genre à partir de faits discursifs, saisis en tant que moments discursifs, permet de mieux appréhender le fonctionnement générique dans son articulation nécessaire à d'autres déterminants indispensables à toute étude discursive dynamique.

- Il convient tout d'abord de situer la notion de genre dans sa relation avec celles de formation discursive. Pêcheux avait établi une relation d'homologie entre formation discursive et idéologie qui projette les frontières socio-historiques sur la matérialité langagière sans introduire de considérations génériques. La notion de genre s'impose pourtant elle-même comme une notion frontière, notion abstraite et théorique qui ne reçoit sa pertinence qu'en relation avec celle de formation discursive, le binôme ainsi constitué n' existant que dans la dynamique de l'interdiscours. Les genres et les formations discursives définissent des espaces d'intelligibilité, théorisés par l'analyste ou intuitifs pour le locuteur, qui n'ont de réalité que dans leurs relations à d'autres espaces construits relationnellement. Autrement dit tout discours les présuppose dans leur pluralité, mais leur délimitation (qui suppose une unité saisie à un moment donné) est niée par l'interprétation qui se joue de leurs frontières. Dans de nombreux travaux, le genre renvoie à l'idée d'une stabilité, voire à celle d'une stabilisation langagière, mais ou cette répétition consiste à identifier des structures régulières et abstraites caractéristiques d'un ensemble de productions - et là, se rencontre sa compréhension la plus fréquente - , ou elle est construite par l'analyste lui-même dans le cadre d'une activité d'interprétation définissant la pertinence des formes - et là, se trouve un des apports essentiels des propositions d'Achard. Dans ce second sens, le genre est corrélé à la notion de registre et de formation discursive, il peut être marqué par une relative hétérogénéité de ses formes, qui, en outre, participent à la caractérisation générique d'autres registres. Ainsi, Achard, fondant son approche sur l'examen de distorsions entre registre et genre, insiste sur l'impossibilité d'établir une typologie générale a priori des genres discursifs et sur « l'absence de critères stables pour appréhender les genres sociaux (le moment, le lieu, le rôle langagier, le 
support... sont autant de critères possibles) » (Branca-Rosoff $1999: 20)$, tout en définissant le genre comme catégorie fondamentalement dynamique et marquée par des régularités formelles. En outre, la notion d'idéologisation introduite par Sarfati permet également de contourner la difficulté portée par la notion de formation discursive, puisqu'elle souligne que l'idéologie est un construit discursif et interdiscursif suscité ou par les caractéristiques génériques, ou par l'hypothèse d'un registre que nous pouvons mettre au compte de la compétence topique de tout locuteur, ou enfin par les deux. Le genre entretient alors une relation dynamique avec les déploiements sémantiques en discours, puisque c'est dans cet espace que se questionne la nature du sens commun, en particulier en tant qu'il peut se comprendre comme une doxa (une manifestation spontanée) ou une idéologie, puisque la généricité peut conditionner l'appréhension d'un élément tel que le jeunisme selon ces deux modalités distinctes, selon que le genre de discours l'oriente vers une caractérisation plus ou moins élaborée.

- Les notions de frontière et de genre sont également interrogeables dans la relation entre langue et contexte, lequel ne saurait être défini de manière restrictive, limité au contexte linguistique ou encore au contexte social, et de manière indépendante l'un de l'autre. Non seulement l'interprétation s'établit à partir de disponibilités de sens, qui ont une existence dans le système clos de la langue, mais celles-ci ne constituent qu'une virtualité pour des formes de «défrontiérisation » qui l'informent par les types de textes et l'interaction des différentes strates sémiotiques. Le sens n'a de réalité que textuelle en fonction de déterminations génériques qui en créent les signifiants dans l'interaction sémantique des composants d'une part, et des jeux intergénériques associés aux hypothèses de registres d'autre part. La notion de sens commun, et sa conception du genre, permet ici d'articuler une prise en compte du caractère social du genre, mais aussi son volet lexical et langagier. En effet, selon Sarfati (2008), "la formation d'un genre est avant tout fonction d'une opération de sémiotisation des normes de la praxis » (Sarfati 2008 : 107), et la topique générique « reçoit sa forme des contraintes que lui impose notamment le site d'énonciation » (ibid. : 100), et consiste en une mise en forme spécifique du savoir d'un groupe.

- Les notions de frontière et de genre enfin s'ouvrent à un questionnement temporel, peu développé dans ce cadre, mais émergent dans notre traitement, en lien avec une historicité notamment déterminée par les objets du débat public à un moment donné. Ces objets existent construits discursivement dans la stabilité d'une contemporanéité, mais le débat public les ouvre pour de nouvelles interprétations évolutives, situées dans l'interdiscursivité et la diachronie. Au-delà de la propriété dynamique du genre, c'est ici son caractère variable qui s'impose : « En dépit de régularités observables, la variation caractérise toute réalisation textuelle. Le facteur principal d'innovation est lié aux conditions pragmatiques de tout acte d'énonciation : la situation d'interaction et les visées et buts des locuteurs contraignent plus ou moins ces derniers à plus ou moins de variations novatrices, de décalages, en jouant tant avec la langue qu'avec les systèmes de genres disponibles » (Adam et Heidmann 2009 : 17). Les auteurs poursuivent en soulignant, comme Achard, que «ces décalages peuvent se transformer en affrontements sur les façons légitimes de parler au sein d'une formation socio-discursive donnée ». Ils se manifestent alors dans le type de fonctionnements polémiques auxquels nous nous sommes intéressés. 


\section{Conclusion}

Nous l'avons vu, en questionnant la notion de frontière dans le but de saisir le sens du point de vue de l'AD, un double fonctionnement est apparu, lié à la conception du sens, et au statut de l'AD. L'analyse des formes linguistiques permet à l'analyste de saisir le passage d'un discours à l'autre, et d'en saisir la complexité notamment au regard de la conflictualité générée par ce passage. En retour, l'AD se constitue comme discipline frontière dans les sciences du langage, et plus largement dans les sciences humaines, puisqu'elle rend possible le passage du sémantique au discursif, du textuel à l'herméneutique, et permet de mesurer les enjeux d'un transfert sociolectal propre au fonctionnement sociodiscursif. Ces transferts peuvent être l'objet de questionnements socio-historiques manifestés par des positionnements métadiscursifs de l'ordre d'une linguistique populaire qui peut constituer une voie d'accès à la plupart des objets du débat public, qui apparaissent comme des espaces de négociation entre clôture et ouverture de frontières. Si le concept de genre n'est pas celui qui est privilégié (à la différence de certaines approches textuelles), il est néanmoins utilisé comme outil pour travailler des relations entre différentes discours, puisque son fonctionnement, et les horizons d'attente qu'il impose parfois, participent du mouvement discursif général. En retour, considéré comme une notion à théoriser, il s'enrichit de la démarche discursive d'une saisie dans la frontière des discours, pour se définir d'une manière peut être plus « souple » que dans d'autres approches, mais aussi peut-être doté d'une certaine efficacité, et richesse, puisqu'il relie des critères d'analyses formels, sociodiscursifs, et pragmatiques.

\section{Bibliographie}

ACHARD, P. (1988) : "La spécificité de l'écrit est-elle d'ordre linguistique ou discursif? », N. Catach éd., Pour une théorie de la langue écrite, Paris, éd. du CNRS.

- (1996) : Rapport Slade (Sociologie du Langage, Analyse de Discours, Énonciation), Présenté au CNRS.

- (1995) : " Formation discursive, dialogisme et sociologie », Langages, $117: 82-96$.

ADAM, J.-M. (2005) : Linguistique textuelle. Des genres de discours aux textes, Paris, Nathan.

ADAM,J.-M. \& BONHOMME, M. (1997) : L'Argumentation publicitaire. Rhétorique de l'éloge et de la persuasion, Paris : Nathan Université.

ADAM, J.-M. et HEIDMANN, U. (2004) : «Des genres à la généricité. L'exemple des contes (Perrault et Les Grimm) », Langages, $153: 62-72$.

- (2009) : Le texte littéraire. Pour une approche interdisciplinaire, LouvainLa-Neuve : Academia-Bruylant. 
AuthIER-Revuz, J. (1982) : « Hétérogénéité montrée et hétérogénéité constitutive : éléments pour une approche de l'autre dans le discours », DRLAV, 26 : 91-151

BEACCO, J.-C. (2004) : « Trois perspectives linguistiques sur la notion de genre discursif », Langages $\mathrm{n}^{\circ} 153: 109-119$.

BRANCA-RosofF, S. (1999) : «Types, modes et genres : entre langue et discours », Langage et société, $87: 5-24$.

Charaudeau, P. (1992) : Grammaire du sens et de l'expression, Paris, Hachette. — (1984) : «Le discours propagandiste », Le français dans le monde, 182 : 100-103.

— (1983) : Langage et discours. Éléments de sémiolinguistique, Paris, Hachette.

FouCAUlt, M. (1969) : L'Archéologie du savoir, Paris, Gallimard.

DiOn, R., FORTIER, F. \& HAGUERAERT, E. éds. (2001) : Enjeux des genres dans les écritures contemporaines, Québec : Nota bene.

GARRIC, N. \& LÉGLISE, I. (2006) : « le discours patronal, discours de propagande », Les Nouvelles journées de l'ERLA : Aspects linguistiques du texte de propagande, Paris, L'Harmattan, 133-146.

GARRIC, N. \& LONGHI, J. (à paraître) : «Atteindre l'interdiscours par la circulation des discours et du sens », soumis à Langage et Société, n²144, juin 2013.

Guilhaumou, J., MALDIDIER, D. et RoBIn, R. (1994) : Discours et archive. Expérimentations en analyse de discours, Liège, Mardaga.

KRIEG-PlANQUE, A. (2004) : «Souligner l'euphémisme : opération savante ou acte d'engagement ? Analyse du « jugement d'euphémisation» dans le discours politique », Semen [En ligne], $17:$ http ://semen.revues.org/2351.

LEEMAN, D. (2004). «L'emploi de juste comme adverbe d'énonciation », Langue française, $142: 17-30$.

Maingueneau, D. (2005) : «L'analyse du discours et ses frontières », Marges linguistiques, $9: 64-75$.

MoIRAND, S. (1992) : «Des choix méthodologiques pour une linguistique de discours comparative », Langages $105: 28-41$.

- (2007) : Les discours de la presse quotidienne. Observer, analyser, comprendre, Paris, PUF, « Linguistique Nouvelle ».

PÊCHEuX, M. (1975) : Les vérités de la Palice, Paris, Maspéro.

RASTIER, F. (2011) : La mesure et le grain. Sémantique de corpus, Paris, Champion.

SARfati, G.-E. (2002) : Précis de Pragmatique, Paris, Nathan.

- (2008) : «Pragmatique linguistique et normativité : remarques sur les modalités discursives du sens commun », Paris, Langages, 170 : 92-108.

— (2010) : «Hermès parmi les loups : sens commun, institutions de sens et doxanalyse ", Au Corps du texte. Hommage à Georges Molinié, Paris, Honoré Champion, Bibliothèque de grammaire et de linguistique, $2010: 339-353$.

- (2011) : " Analyse du discours et sens commun : institutions de sens, communautés de sens, doxa et idéologie », Guilhaumou J. \& Schepens P. (dir.), Matériaux philosophiques pour l'analyse du discours, Presses Universitaires de Franche-Comté : 139-174.

TLFI : Trésor de la Langue Française Informatisé, disponible sur http ://atilf.atilf.fr/ 